\title{
Retrospective analysis of metastatic sarcoma patients
}

\author{
TOMOKI NAKAMURA, AKIHIKO MATSUMINE, TAKAO MATSUBARA, \\ KUNIHIRO ASAMUMA, RUI NIIMI, ATSUMASA UCHIDA and AKIHIRO SUDO \\ Department of Orthopaedic Surgery, Mie University Graduate School of Medicine, Mie 514-8507, Japan
}

Received November 26, 2010; Accepted December 23, 2010

DOI: $10.3892 / \mathrm{ol} .2011 .238$

\begin{abstract}
Numerous studies have reported the survival of metastatic sarcoma patients who have undergone either a lung metastasectomy or chemotherapy. However, little is known with regards to the clinical course of patients with bone or soft tissue sarcomas who have succumbed to disease. This study aimed to analyze the metastatic patterns of sarcoma patients and to describe the clinical course after the detection of distant metastasis. We reviewed the clinical records of 255 patients with a diagnosis of sarcoma who were referred to our institution, and found 63 patients who succumbed due to metastasis. We examined the clinical features of the initially detected distant metastases, the subsequent clinical course up to the time of patient death and the survival time of patients who died of lung metastasis. Of the 63 patients who died of distant metastasis, $52(83 \%)$ developed lung metastasis as the first metastatic site, while $22(35 \%)$ developed extra-pulmonary metastasis. The majority (77\%; 49 of 63 patients) died of primary metastasis. While all 18 bone sarcoma patients died of lung metastasis, 11 of the 45 soft tissue sarcoma patients died of extra-pulmonary metastasis. Six patients died of brain metastasis. The survival of the patients with lung metastasis was only approximately 6 months following the cessation of treatment, regardless of the type of treatment used. These results indicate that planned follow-up and treatment of sarcomas require a precise knowledge of tumor clinical behavior, particularly of the preponderant activity.
\end{abstract}

\section{Introduction}

Although the prognosis for patients with bone or soft tissue sarcomas has shown marked improvement over the past three decades, those who develop local recurrence or metastatic disease continue to have high mortality rates. Of all patients diagnosed with malignant musculoskeletal tumors, 5-30\% (1-3) have a recurrence and $10-38 \%$ of patients present with

Correspondence to: Dr Akihiko Matsumine, Department of Orthopaedic Surgery, Mie University Graduate School of Medicine, Edobashi 2-174, Tsu, Mie 514-8507, Japan

E-mail: matsumin@clin.medic.mie-u.ac.jp

Key words: cause of death, metastasis, sarcoma clinically detectable metastases (4-6). A number of studies have reported the survival of patients who have undergone either a lung metastasectomy or chemotherapy (4,5,7-9).However, little is known about the clinical course of patients with bone or soft tissue sarcomas who have succumbed to the disease. For example, only a few reports are available regarding the clinical course, such as the metastatic site, which led to the cause of death or the survival time following initial detection of metastases (10). Thus, this study aimed to analyze the metastatic patterns of sarcoma patients and to describe the clinical course following the detection of distant metastasis.

\section{Patients and methods}

We retrospectively reviewed the medical records of newly diagnosed patients with either bone or soft tissue sarcoma referred to Mie University Hospital between January 1999 and December 2008. Well-differentiated liposarcomas were excluded from this study. During this period, 255 patients with a diagnosis of sarcoma were referred to our institution. We reviewed the clinical records of these patients and found 63 patients who died of metastasis.

We examined the clinical features of the initially detected distant metastases, the subsequent clinical course up to the patient's death and the survival time of the patients who succumbed to lung metastasis.

Statistical analysis. Overall survival was estimated using the Kaplan-Meier method. Factors affecting patient survival were examined based on the log-rank test. The StatMate III program (ATMS Co., Tokyo, Japan software) was used to conduct statistical analysis. Post-metastatic survival was defined as the duration between the first detection of distant metastases and patient death. The post-treatment survival time was defined as the duration between the final treatment [surgery, lung radiofrequency $(\mathrm{RF})$ ablation and chemotherapy] for metastases and death.

\section{Results}

Patient and tumor characteristics and follow-up. A total of 34 male and 29 female patients were included in our study. The average age was 51 years (range 6-80), including 17 patients who were $\geq 70$ years of age. The follow-up periods ranged from 1 to 82 months (average 16) after the initial detection of metastasis 
The histological diagnoses of the primary bone tumor were osteosarcoma $(\mathrm{n}=10)$, malignant fibrous histiocytoma (MFH) of the bone $(n=3)$, chondrosarcoma $(n=2)$, Ewing's sarcoma $(n=2)$ and others $(n=2)$. Soft tissue tumor included malignant peripheral nerve sheath tumors $(n=9), M F H(n=9)$, leiomyosarcoma $(n=6)$, myxoid liposarcoma $(n=4)$, synovial sarcoma $(\mathrm{n}=3)$, alveolar soft part tumor (ASPS) $(\mathrm{n}=3)$, extra-skeletal myxoid chondrosarcoma $(n=2)$, epithelioid sarcoma $(n=2)$ and others $(\mathrm{n}=6)$. According to the American Joint Commission on Cancer classification and stage grouping of bone sarcomas, 9 were classified as stage $2 \mathrm{~B}, 6$ as $3 \mathrm{~A}$ and 1 as stage $3 \mathrm{~B}$. Of the soft tissue sarcomas, 3 were classified as stage 2, 26 as stage 3 and 18 as stage 4.

Post-treatment follow-up was performed at 3-month intervals for 2 years, then every 4-6 months for 5 years, and every year subsequently. At follow-up, a chest X-ray was routinely carried out, and an examination using computed tomography (CT) (X-Vigor or Aquilion; Toshiba, Tokyo, Japan; or HiSpeed Advantage Qx/I; GE Healthcare, USA; or Asteion; Toshiba) was performed at 3- to 6-month intervals to detect distant metastasis. A CT scan of the abdomen and pelvic cavity was performed at 6-month intervals for the patients with myxoid liposarcoma.

Treatment of the primary tumor. A surgical resection was performed in 56 of the 63 patients. Local recurrence occurred in 26 of the 56 patients. In 10 of the 26 patients, the local recurrence had developed prior to the first detection of distant metastasis. Local recurrence was treated with surgical resection $(n=20)$, radiotherapy $(n=4)$ and chemotherapy $(n=3)$. In total, 7 of the 63 patients were not treated with surgery for their primary tumor. Four patients underwent radiotherapy due to their advanced age $(\mathrm{n}=2)$, or an unresectable tumor size and location $(n=2)$. One patient with post-radiation MFH at a sacro-iliac lesion underwent carbon ion radiotherapy since it was considered to be less invasive. One patient received only palliative therapy for multiple metastatic lesions. A 6-year-old female with osteosarcoma in her femur was treated only with chemotherapy as her family rejected surgical excision.

Clinical features of the initially detected distant metastases and the clinical course until patient death. The location of the initially detected distant metastases was investigated. In 53 of the 63 patients, lung metastasis developed (83\%) and in 21 patients (33\%) extra-pulmonary metastasis developed. The average size of the maximum diameter of the lung metastases was $10 \mathrm{~mm}(3-40 \mathrm{~mm})$, and the average number of lung metastases was 5 (1-20) upon detection.

The sites of extra-pulmonary metastasis were bone $(\mathrm{n}=10)$, liver $(n=5)$, soft tissue $(n=3)$, brain $(n=2)$, heart $(n=2)$, pelvic cavity $(\mathrm{n}=1)$ and stomach $(\mathrm{n}=1)$. The occurrence of lung and extra-pulmonary metastases was observed in 11 patients. Ten patients had only extra-pulmonary metastasis without any co-existing lung metastasis in the following sites: bone $(n=5)$, liver $(n=1)$, brain $(n=1)$, heart $(n=1)$, subcutaneous tissue $(n=1)$ and pelvic cavity $(\mathrm{n}=1)$ (Fig. 1).

Of the 53 patients who had lung lesions at the first detection of distant metastasis, 46 died of progression of lung metastasis, 2 of liver metastasis, 4 of brain metastasis and 1 of adrenal metastasis. Of the 5 patients who had bone lesions at the first

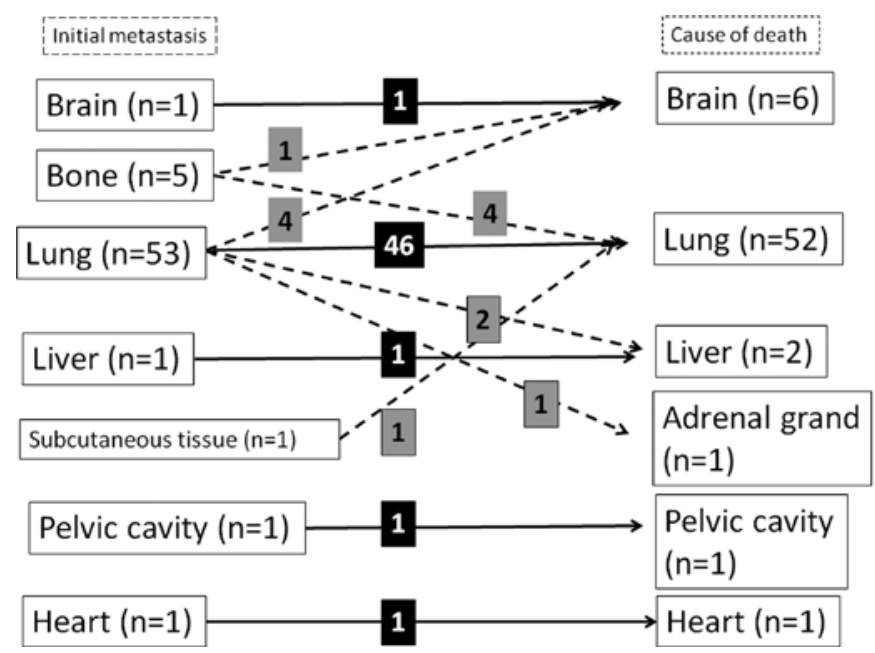

Figure 1. Relationship between the primary metastatic site and the cause of death: left section, initial metastatic site; right section, focus with cause of death.

detection of distant metastasis, 4 died of lung metastasis and 1 of brain metastasis. One patient who had distant metastasis in the subcutaneous tissue at the first detection of metastasis died of lung metastasis. Each patient who had metastasis in the brain, liver, heart or pelvic cavity at the first detection of the metastasis died of aggravation of the initial metastasis (Fig. 1). As a result, of the 63 patients, 52 died of lung metastasis, 6 of brain, 2 of liver and 1 of heart, pelvic cavity or adrenal metastasis. A total of $77 \%$ of patients ( 49 of the 63 ) succumbed to the primary metastasis.

While all 18 bone sarcoma patients died of lung metastasis, 11 of the 45 soft tissue sarcoma patients succumbed to extrapulmonary metastasis. Seven of these 11 patients died of other additional distant metastases. The 3 patients with ASPS died of brain metastases.

The 10 patients that survived $>2$ years following the initial detection of distant metastasis developed other additional distant metastases (Table I). The sites of additional distant metastasis were: retroperitoneum $(n=2)$, bone $(n=2)$, soft tissue $(n=1)$, lymph node $(n=1)$, radial nerve $(n=1)$, lung $(n=1)$, adrenal grand $(\mathrm{n}=1)$ and brain $(\mathrm{n}=1)$.

Length of survival of the 52 patients who succumbed to lung metastasis. The length of survival of the 52 patients who died of lung metastasis was examined. The median post-treatment survival of the 20 patients who underwent treatment for metastasis, including metastasectomy, RF ablation and chemotherapy, was 6 months (Fig. 2). The following treatments for the lung metastases were performed: lung RF ablation alone $(n=5)$, lung RF ablation and chemotherapy $(n=6)$, metastasectomy and chemotherapy $(n=4)$, chemotherapy alone $(n=4)$, and metastasectomy and lung RF ablation ( $\mathrm{n}=1)$.

The median post-metastatic survival of the 32 patients who received no treatment for their metastasis was 7.2 months. By contrast, the median post-metastatic survival in the 20 patients who underwent treatment for metastasis was 16 months. This represents a significant difference in the survival rate $(\mathrm{P}=0.003)$ (Fig. 3). 
Table I. Pattern of metastases in patients who survived more than 2 years following the initial metastasis.

\begin{tabular}{lcccc}
\hline Diagnosis & $\begin{array}{c}\text { Initial metastatic } \\
\text { site }\end{array}$ & $\begin{array}{c}\text { Other metastatic } \\
\text { site }\end{array}$ & $\begin{array}{c}\text { Cause of } \\
\text { death }\end{array}$ & $\begin{array}{c}\text { Follow-up periods } \\
\text { (months) }\end{array}$ \\
\hline ASPS & Lung & Brain & Brain & 45 \\
Synovial sarcoma & Lung & Adrenal gland & Adrenal gland & 32 \\
Ewing's sarcoma & Lung & Retroperitoneum & Lung & 85 \\
MGCT & Lung & Soft tissue & Lung & 63 \\
MFH & Subcutaneous & Lung & Lung & 43 \\
ESCS & Lung & Lymph node & Lung & 59 \\
Synovial sarcoma & Lung & Radial nerve & Lung & 29 \\
Myxoid sarcoma & Lung & Retroperitoneum & Lung & 25 \\
Osteosarcoma & Lung & Bone & Lung & 35 \\
Rhabdomyosarcoma & Lung & Brain & Brain & 24 \\
\hline
\end{tabular}

ASPS, alveolar soft part sarcoma; MGCT, malignant granular cell tumor; MFH, malignant fibrous histiocytoma; ESCS, extra-skeletal chondrosarcoma.

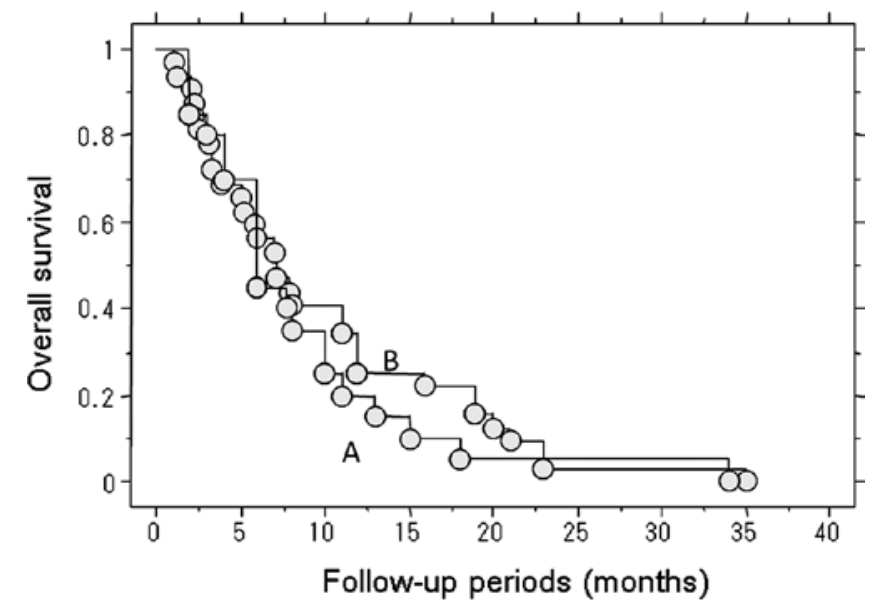

Figure 2. Kaplan-Meier curves showing the post-treatment survival of 20 patients receiving treatment for the metastasis (A), and the postmetastatic survival of 32 patients who did not receive any treatment for the metastasis (B)

\section{Discussion}

Regarding the location of metastases, $62-82 \%$ of sarcoma patients had lung metastasis and 50-70\% had isolated lung metastases in previous studies $(4,11)$. In our series, $83 \%$ (52 of 63 patients) developed lung metastasis as the initial metastatic site. Therefore, the present results are consistent with previous reports. Although numerous reports exist regarding the initial metastatic site in sarcoma patients and the treatment of patients with metastasis, few reports are available regarding the location of the metastatic lesion that ultimately caused patient death (4,5,7-11). In the present study, $82.5 \%$ of patients succumbed to lung metastasis and $10 \%$ to brain metastasis. Other patients succumbed to liver (3\%), heart (1.5\%), adrenal grand $(1.5 \%)$ and pelvic metastasis (1.5\%).

Brain metastases from soft tissue sarcoma are believed to occur in a minority of soft tissue sarcoma patients, with a reported prevalence ranging between 1 and $6 \%(12,13)$. In

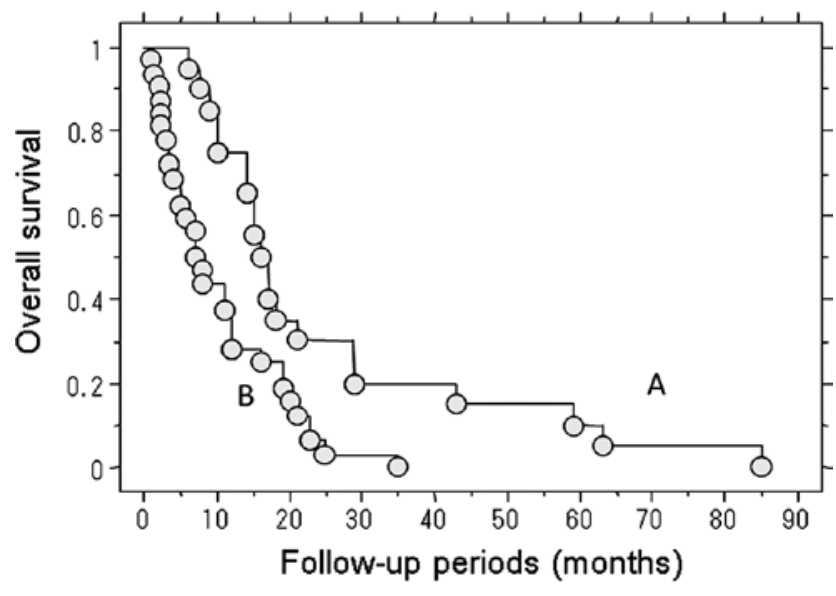

Figure 3. Kaplan-Meier curves showing post-metastatic survival in patients who received treatment (A) and patients who did not receive any radical treatment (B).

osteosarcoma, the incidence of brain metastasis is reported to be $2-6.5 \%$ (14). Particularly in the absence of lung metastases, brain metastasis is thought to be a relatively uncommon event in the natural history of bone and soft tissue sarcomas. In our cases, only 1 patient with epithelioid sarcoma had brain metastasis without lung metastasis. ASPS has been reported to metastasize to the brain more commonly than other types of high-grade sarcoma, although the majority of studies concerning ASPS are in the form of case reports and small corrective series (15-17). In our present series, brain metastasis developed within 4 years of the primary surgery in the 3 patients with APSP. We therefore recommend the regular use of intracranial CT imaging for patients with ASPS.

Although the prognosis of sarcoma patients has improved, extra-pulmonary metastases should be further investigated. In our series, 11 of the 45 soft tissue sarcoma patients died of extra-pulmonary metastasis. In addition, 7 of the 11 patients succumbed to other additional distant metastases. Although follow-up chest $\mathrm{CT}$ and physical examinations were routinely 
performed at 3- to 6-month intervals to detect distant metastasis, it was difficult to identify new extra-pulmonary metastatic lesions without clinical symptoms. Therefore, we recommend regular screening using a contrast-enhanced CT scan to detect abdominal or pelvic metastases at 3- to 6-month intervals, as well as chest CT scans, particularly for soft tissue sarcoma patients, even when no clinical symptoms occur.

Few reports are available on the metastatic site causing patient death and the relationship between the metastatic site and the duration of survival after final treatment (surgery, lung RF ablation, chemotherapy and radiation) for sarcoma (10). In the present study, the median post-metastatic survival in the 32 patients who did not receive radical treatment for metastases was 7.2 months. The median post-treatment survival in the 20 patients who received treatment for the metastatic disease was 6 months. This suggests that the survival of patients with lung metastases is approximately 6 months in the absence of treatment, regardless of prior treatments. One limitation of the present study is that it included various different histological diagnoses which exhibit different clinical behavior. Therefore, further large-scale investigations are required to clarify the effects of the metastatic site on survival.

This study provides crucial data regarding the clinical course of metastatic sarcoma patients. Of the 63 patients who died of distant metastasis, 52 (83\%) developed lung metastasis as the initial metastatic site, while $22(35 \%)$ developed extrapulmonary metastasis. A total of 51 patients succumbed to lung metastasis, 6 to brain, 3 to liver and 1 to heart, pelvic cavity and adrenal metastasis. The majority (77\%; 49 of 63 patients) died of the primary metastasis. Six patients $(10 \%)$ died of brain metastasis, while 11 (24\%) of the 45 soft tissue sarcoma patients died of extra-pulmonary metastasis. It is therefore crucial to pay particular attention to the appearance of brain metastasis during treatment. In addition, we recommend that routine screening using contrast-enhanced CT scans be utilized to detect abdominal or pelvic metastases. Chest CT scans should also be performed on a regular basis, even when no clinical symptoms are noted in patients.

In conclusion, the survival of patients with lung metastases is only approximately 6 months following the cessation of treatment, regardless of the type of treatment initially used.

\section{References}

1. Weiss SW and Goldblum JR: Local recurrence. In: Enzinger and Weiss's Soft Tissue Tumors. 4th edition. Mosby, St. Louis, pp28-32, 2001.

2. Zager GK, Ballo MT, Pesters PW, et al: Prognostic factors for patients with localized soft-tissue sarcoma treated with conservation surgery and radiation therapy; an analysis of 1225 patients. Cancer 97: 2530-2543, 2003.

3. Duffaud F, Digue L, Mercier C, et al: Recurrence following primary osteosarcoma in adolescents and adults previously treated with chemotherapy. Eur J Cancer 39: 2050-2057, 2003.

4. Kane JM III, Finley JW, Driscoll D, et al: The treatment and outcome of patients with soft tissue sarcomas and synchronous metastases. Sarcoma 6: 69-73, 2002.

5. Harting MT, Blakely ML and Jaffe N: Long-term survival after aggressive resection of pulmonary metastases among children and adolescents with osteosarcoma. J Pediatr Surg 41: 194-199, 2006.

6. Pollock RE, Karnell LH and Menck HR: The national cancer data base report on soft tissue sarcoma. Cancer 78: 2247-2257, 1996.

7. Weiser MR, Downey RJ and Leung DHY: Repeat resection of pulmonary metastases in patients with soft-tissue sarcoma. J Am Coll Surg 191: 184-190, 2000.

8. Karavasilis V, Seddon BM, Ashley S, et al: Significant clinical benefit of first line palliative chemotherapy in advanced softtissue sarcoma. Cancer 112: 1585-1591, 2008

9. D'Adamo DR, Anderson SE and Albritton K: Phase III study of doxorubicin and bevacizumab for patients with metastatic softtissue sarcomas. J Clin Oncol 23: 7135-7142, 2006.

10. Jeffree GM, Price CHG and Sissons HA: The metastatic patterns of osteosarcoma. Br J Cancer 32: 87-107, 1975.

11. Vezeridis MP, Moore R and Larakousis CP: Metastatic patterns in soft tissue sarcomas. Arch Surg Oncol 118: 915-918, 1998.

12. Espat NJ, Bilsky M, Lewis JJ, et al: Soft tissue sarcoma brain metastases. Cancer 94: 2706-2711, 2002.

13. Gupta T, Laskar S, Gujral S, et al: Brain metastases in soft tissue sarcomas: case report and literature review. Sarcoma 9: 147-150, 2005.

14. Yonemoto $\mathrm{T}$, Tatezaki S, Ishii T, et al: Longterm survival after surgical removal of solitary brain metastasis from osteosarcoma. Int J Clin Oncol 8: 340-342, 2003.

15. Evans HL: Alveolar soft-part sarcoma. A study of 13 typical examples and one with a histologically atypical component. Cancer 55: 912-917, 1985.

16. Temple HT, Scully SP, O'Keefe RJ, et al: Clinical presentation of alveolar soft-part sarcoma. Clin Orthop 300: 213-218, 1994.

17. Portera CA Jr, Ho V, Patel SR, et al: Alveolar soft part sarcoma: clinical course and patients of metastasis in 70 patients treated at a single institution. Cancer 91: 585-591, 2001. 